34

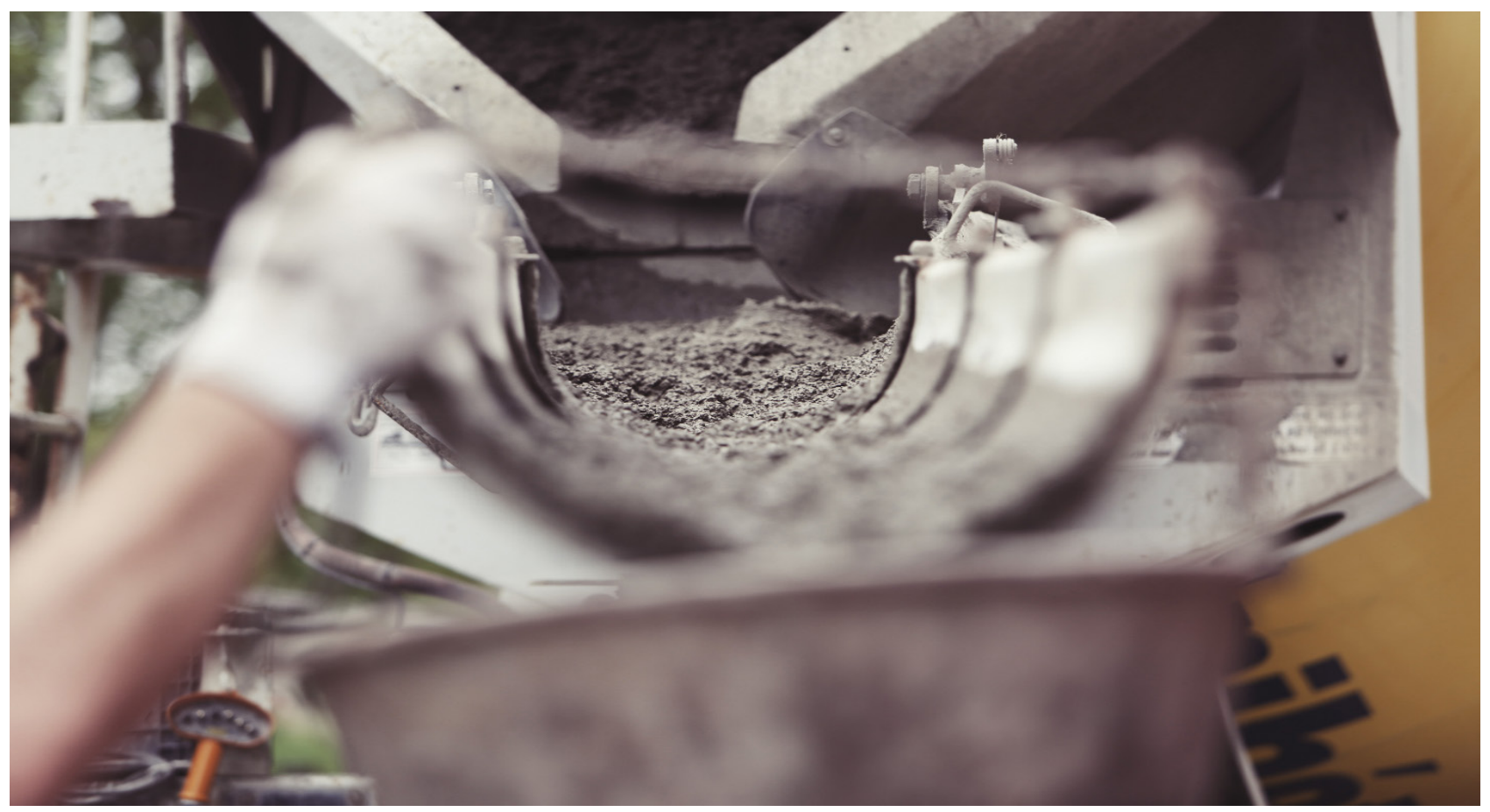

\title{
Propuesta para el diseño de cemento alternativo basado en residuos de ladrillo de arcilla roja activados alcalinamente
}

\section{Proposal for alternative cement design based on alkali activated red clay brick waste}

Santiago Celis-Vergel

Ingeniero Civil, santiagocv@ufps.edu.co,

https://orcid.org/ 0000-0002-1344-3180, Universidad Francisco de

Paula Santander, Cucuta, Colombia. 


\section{Resumen}

Los cambios constantes que han sufrido los materiales en la construcción se apegan a necesidades de su contexto, es por ello que el concreto es uno de los materiales más importantes para las obras civiles, y su evolución es primordial para la mejora continua y poder ayudar a la comunidad en aprovechar sus beneficios, es por ello que el propósito de esta investigación que se formula desde su primera etapa es poder proponer un concreto a base de los diferentes residuos de los ladrillo de arcilla para la realización de pruebas de laboratorio y así poder saber cómo se comporta el concreto cuando se le añade este material reciclado, con esto validar los resultados que se pudieran aprovechar en varios contextos y así disminuir o mejorar características que ayudan a elevar los costos de producción de este material.

Palabras clave: Arcilla roja, cemento, ladrillos, residuos.

\section{Abstract}

The constant changes that construction materials have undergone are in keeping with the needs of their context, which is why concrete is one of the most important materials for civil works, and its evolution is essential for continuous improvement and to help the community to take advantage of its benefits, That is why the purpose of this research, which is formulated from the first stage, is to be able to propose a concrete based on the different clay brick wastes for laboratory testing and thus be able to know how the concrete behaves when this recycled material is added, thus validating the results that could be used in various contexts and thus decrease or improve characteristics that help to increase the production costs of this material.

Keywords: Red clay, cement, bricks, waste. 


\section{Introducción}

Se dice que la ciudad del futuro no solo debe tenerbajosconsumos de energíayemisiones de gases invernadero. Sino que debe tender hacia políticas de cero residuos, lo que contribuirá para un desarrollo sostenible y a reducir la huella de carbono. Muchos desperdicios, incluidos los de minería, siderurgia, construcción demolición, pueden ser utilizados como materias primas en otras industrias. Las principales barreras para este tipo de reciclaje son las calidades de estos desperdicios, en especial sus características físicas y químicas (las cuales son muy heterogéneas), y el bajo costo o alta disponibilidad de materias primas tradicionales. Por eso, la activación alcalina y geo-polimerización son tecnologías que nos permiten utilizar algunos residuos que son inservibles en otras industrias [1].

Cabe anotar que la aplicación de estas tecnologías en la producción de cementos, en adición a promover el consumo de desperdicios como materia prima, tiene beneficios adicionales como el bajo consumo energético y reduce el nivel de emisiones, el cual ha sido demostrado en varios estudios es mayor en el cemento portland tradicional [2].

Los residuos cerámicos son generados en la industria de la construcción y en la industria cerámica en general en su proceso de producción. Aproximadamente $45 \%$ de los residuos de construcción y demolición son residuos de tipo cerámico, como ladrillos, tejas o porcelana. Según algunos estudios, la industria cerámica europea genera un volumen de desperdicio equivalente al $3-7 \%$ del total de su producción, lo que indica que millones de toneladas de arcilla al año son depositadas en depósitos y rellenos sanitarios con un mínimo o nulo porcentaje de reutilización. Actualmente los países que más residuos de construcción y demolición (RCD) son China, Estados Unidos y los países de la Unión Europea, generando aproximadamente 606 millones de toneladas al año, sin embargo esta cantidad puede ser aún mayor debido a desastres naturales [3].

EnpaísescomoAlemania, DinamarcayHolanda, el porcentaje de reutilización de estos residuos alcanza hasta un $80 \%$ mientras que el promedio en el resto de países no alcanza al 30\%. Sin embargo en Colombia no hay disponibilidad de cifras oficiales al respecto, algunos estudios indican que hasta 12 millones de toneladas de RCD son generadas al ano en ciudades de altos crecimientos demográficos como Bogotá y son completamente inutilizadas [4].

En Norte de Santander, en se cuenta con la arcilla de mejor calidad del mundo, ésta industria tiene una producción aproximada para 2017 de 188.433 toneladas anuales, de las cuales por lo menos un $5 \%$ son desechadas o descartadas en su proceso de control de calidad, lo cual supone ya un aproximado de 12.255 toneladas de residuos cerámicos que no se están reutilizando. La gran mayoría de estos residuos terminaran en rellenos que no cumplen con la normativa necesaria, o en el peor de los casos, en botaderos ilegales en la rivera del rio, contaminando asi nuestra principal fuente hídrica [5]. Es por esto que se hace necesario buscar una solución que reduzca la huella ambiental de una industria tan importante para la región como lo es la cerámica y darle un valor agregado a estos residuos, no solo ambientalmente hablando sino económicamente, generando una oportunidad para la creación de nuevas empresas y por ende nuevos puestos de trabajo.

Esta investigación se enfoca en el diseño de una propuestra encamindada a la reutilización de los residuos de la industria cerámica en particular, de la cual el residuo de los ladrillos o tejas de arcilla roja RCBW (por sus siglas en inglés) pueden ser activadas alcalinamente para crear cementos cuyas resistencia a la 
compresión llegan incluso a superar las del cemento portland tradicional.

\section{Objetivo}

Diseñar una propuesta para reducir el impacto ambiental generado en la industria de la arcilla de la región a través de la producción de un cemento sostenible como alternativa al cemento portland tradicional.

\section{Marco teórico}

Los Geopolimeros se refieren en muchas ocasiones como un subconjunto de materiales activados alcalinamente. Las cenizas volantes con un bajo contenido en calcio y arcillas calcinadas (ej. metacaolin) son los precursores más comunes utilizados en la síntesis del geopolímero [6].

El cemento Portland es considerado el material de construcción por excelencia ya que, a día de hoy, no hay ningún conglomerante que posea la aceptación que posee este material. Esto es debido, principalmente, a sus buenas prestaciones, a su buena relación calidad/ precio y a que las materias primas a partir de las cuales se fabrica pueden encontrarse prácticamente en todo el mundo. Sin embargo, el desarrollo de cementos alternativos al cemento Portland que se obtengan a través de procesos que supongan menores emisiones de gases contaminantes a la atmósfera y con un apreciado ahorro energético, constituye una línea de investigación prioritaria y de gran interés a escala mundial [6].

Dentro de estos materiales alternativos se encuentran aquellos que proceden de la activación alcalina de aluminosilicatos naturales (arcillas) y/o subproductos industriales, como son las escorias de alto horno o las cenizas volantes. Estos cementos y hormigones activados alcalinamente se caracterizan por presentarbajoscalores dehidratación, elevadas prestaciones mecánicas y buena durabilidad frente a diferentes agresivos químicos y físicos, además de no requerir en su elaboración los elevados consumos energéticos que son inherentes al proceso de fabricación de los cementos Portland. Sin embargo, aún no se puede decir o establecer que los cementos alcalinos se basan en una química limpia con el medioambiente, ya que los procesos de producción de las disoluciones alcalinas tales como los silicatos sódicos, emiten grandes cantidades de $\mathrm{CO}_{2}$ a la atmósfera además de ser un proceso costoso [6].

El Material activado alcalinamente abarca esencialmente cualquier sistema aglutinante obtenido por reacción de una fuente de metal alcalino (sólido o disuelto) con un sólido en polvo de silicato. Estos sólidos pueden ser aluminosilicatos ricos o pobres en calcio, tales como las escorias de alto horno o las cenizas volantes, respectivamente. Lasfuentesalcalinas empleadas pueden incluir hidróxidos alcalinos, silicatos, carbonatos, sulfatos, aluminatos u óxidos, esencialmente cualquier sustancia soluble que puede suministrar cationes de metales alcalinos, elevar el pH de la mezcla de reacción y acelerar la disolución del precursor sólido [7].

- Ordinary Portland Cement (OPC): Cemento Portland Ordinario

- Red Clay Brick Waste (RCBW): Desecho de ladrillo de arcilla roja

- Residuos de Construcción y Demolición(RCD)

Una activación alcalina de distintos residuos cerámicos con una mezcla de hidróxido de sodio $(\mathrm{NaOH})$ y silicato de sodio $\left(\mathrm{Na}_{2} \mathrm{SiO}_{3}\right)$ en concentración de $6 \mathrm{M}$ y reporto una resistencia a la compresión de $13 \mathrm{MPa}$. Ellos le atribuyeron esta relativamente baja resistencia a la s0065mi-cristalina naturaleza de este tipo de materias primas [8-9]. 
Autores como [3] llevaron a cabo una activación alcalina de residuos de ladrillos de construcción con una mezcla de $\mathrm{NaOH} \mathrm{Y}$ $\mathrm{Na}_{2} \mathrm{SiO}_{3}$ a una razón de $8 \% \mathrm{Na}_{2} \mathrm{O}$ con respecto a su precursor y obtuvo una resistencia de 40 MPa después de 28 dias de curado [4]. En otro estudio posterior su autor reporto una resistencia de $50 \mathrm{MPa}$ usando una mezcla de $60 \%$ residuo de concreto y $40 \%$ de residuo de ladrillo activados con $\mathrm{NaOH}$ y $\mathrm{Na}_{2} \mathrm{SiO}_{3}$ en solución 1:4 como el predecesor y el mismo porcentaje de $\mathrm{Na}_{2} \mathrm{O}$ en la mezcla [8-9].

Materiales y métodos

Esta investigación es de carácter Descriptivo, considerando que se busca diseñar una propuesta que permita desde el punto de vista experimental, comprobar la resistencia a la compresión del mortero utilizando como variables las distintas dosificaciones de mezcla que se ensayaran para finalmente analizar los resultados e identificar la más óptima.

Resultados

Diseño de la propuesta

\section{Alcance}

Se va a enfocar únicamente en los residuos de piezas dearcillarojaRCBW(red claybrickwaste) como lo son ladrillos y tejas tradicionales. Por ahora no se trataran piezas cerámicas más elaboradas como la porcelana o ninguna pieza con cualquier tipo de recubrimiento especial.

Adicionalmente el único parámetro a considerar para las mediciones y el análisis de resultados será la resistencia a la compresión. No será evaluada ninguna otra característica de resistencia mecánica ni química ni tampoco se realizara un análisis de precios unitarios.
Ambito de aplicación

El ámbito macro de la investigación se que se define corresponde a todas las ladrilleras y tejares de Norte de Santander, o en general a toda la industria cerámica de la región, pues es a partir de los residuos generados en la producción de esta industria que se desarrollara el mortero en el laboratorio.

El ámbito micro u objeto de estudio se enfoca en los residuos de tres tejares representativos de la región. Estos son: Margres, Casa Blanca y el Tejar Santa Rosa.

\section{Delimitaciones}

- Delimitación espacial: El experimento se llevara a cabo en el campus de la Universidad Francisco de Paula Santander en la ciudad de Cúcuta, Norte de Santander. de igual forma, la recolección de los residuos cerámicos a utilizar en las pruebas serán donados por tres empresas cerámicas distintas, todas ubicadas en Norte de Santander.

- Delimitación Conceptual:

- Red Clay Brick Waste (RCBW): Desecho de ladrillo de arcilla roja

- Residuos de Construcción y Demolición(RCD)

- Ordinary Portland Cement (OPC): Cemento

Portland Ordinario

- Material activado alcalinamente

- Geopolímero

- Resistencia a la compression

Fases

Fase1. Definir las distintas dosificaciones que se ensayaran, basándose en las descritas en antiguas investigaciones.

Fase 2. Preparar morteros utilizando las dosificaciones escogidas. 
Fase 3. Ensayar los morteros evaluando su resistencia a la compresión.

Fase 4. Comparar los resultados obtenidos y analizar cuál sería la dosificación óptima.

\section{Materia prima}

La materia prima utilizada para la fabricación de cementos híbridos alcalinamente activados son RCBW y OPC respectivamente. EI RCBW será seleccionado de unas pilas de residuos en tres tejares de la región. Este desperdicio es generado en el proceso de producción del tejar pues los ladrillos y tejas se parten en el proceso de secado y transporte del material. Para el proceso de triturado y pulverización del RCBW se necesita un molino de bola. Adicionalmente, un análisis químico molecular y un análisis granulométrico laser sería ideal para realizar las pruebas pero esta opción está sujeta a disponibilidad de equipos en la región y al presupuesto aprobado para esta investigación [3].

Para la evaluación de las propiedades mecánicas y micro estructurales de RCBW cuando es activada con $\mathrm{NaOH}$ y $\mathrm{Na}_{2} \mathrm{SiO}_{3}$ para obtener cemento activado alcalinamente (100\% RCBW) y un cemento hibrido (RCBW + Cemento Portlant Ordinario (OPC)). RCBW será suministrado por un tejar de la región. El efecto en la resistencia a la compresión de las siguientes variables será analizado: $\mathrm{Na}_{2} \mathrm{O}$ $/ \mathrm{SiO}_{2}$ y $\mathrm{SiO}_{2} / \mathrm{Al}_{2} \mathrm{O}_{3}$ en relación molar, tipo de curado (Temperatura ambiente a $25 \mathrm{C}$ y un curado térmico a $70 \mathrm{C}$ por 24 y 48 horas) y cantidad de OPC (entre 5 y $20 \%$ del peso respecto al contenido de RCBW) [10-11].

Las pastas alcalinas activadas simples (100\% RCBW) e híbridas (RCBW + OPC) se prepararán en condiciones equivalentes. El OPC se incorporará en concentraciones entre 5 y $20 \%$ en peso con respecto a la RCBW. Como activadores alcalinos, $\mathrm{NaOH}$ (98.22\% pureza) y silicato de sodio comercial
$\left(\mathrm{Na}_{2} \mathrm{SiO}_{3} \mathrm{xH}_{2} \mathrm{O}\right)\left(\mathrm{SiO}_{2}=32.09 \%, \mathrm{Na}_{2} \mathrm{O}=\right.$ $11.92 \%$ y $\mathrm{H}_{2} \mathrm{O}=55.99 \%$ ) serán empleados. La relación liquido - solido ( $L$ / S) será de 0.25 asegurando una trabajabilidad adecuada [12]

La mezcla se llevará a cabo en varias etapas, de la siguiente manera: (1) preparación de la solución activadora en las proporciones requeridas, (2) mezcla del solución activadora con el sólido (RCBW) o mezcla de sólidos (OPC + RCBW) previamente homogeneizado y luego adición del agua restante para completar la relación L/S especificada. Las pastas activadas alcalinamente se obtendrán usando un mezclador industrial por tiempos de 5 minutos. Estas pastas aun en estado fresco, se moldearan en formaletas cubicas de $5 \mathrm{~cm}$ de arista y se harán vibrar por 30 segundos en una cama vibradora para eliminar el exceso de aire atrapado en el interior y asi evitar porosidades altas. Entonces, los moldes serán cubiertos con una película de polietileno que evita la evaporación del agua de mezcla libre y curada a temperatura ambiente (25 C) y 70 C por 24 y $48 \mathrm{~h}$. Después de este período, los cubos de prueba serán llevados a una cámara de curado a una humedad relativa cercana al $80 \%$ hasta alcanzar la edad de prueba. Finalmente se evaluara la resistencia a la compresión de cada cubo en una maquina universal. En cada caso seemplearán tres especímenes. Los resultados serán graficados y analizados posteriormente [13-14].

\section{Referencias}

[1] Comunidad Madrid. "Estrategia de Gestión Sostenible de los residuos de la Comunidad de Madrid", 2017. [En línea]. Disponible en: https://www.comunidad.madrid/sites/default/ files/doc/medio-ambiente/f_residuos_cm contdc_con_marcadores.pdf

[2] M.A. Sanabria Ramírez, "Propuesta de mejora para el proceso productivo de la empresa cementos Tequendama". Universidad Católica de Colombia, Bogotá, 2018 
[3] R. Robayo-Salazar, R. Mejía de Gutiérrez y A. Mulford-Carvajal, "Producción de elementos constructivos a partir de residuos de ladrillo activados alcalinamente", Facultad de Ingeniería, vol. 25, núm. 43, pp. 21-30, 2016.

[4] Inerco, Consultoría Colombia, "Valoración energéticaderesiduos:proyecto:WTEColombia, 2018. [En línea]. Disponible en: https:// bdigital.upme.gov.co/bitstream/001/1339/5/ Productos $\% 201 \% 2 \mathrm{C} 2 \% 20 \mathrm{y} \% 203 \_V 2 . p d f$

[5] A. García Gutiérrez y A. Forero Gamboa, "Plan estratégico para mejorar la competitividad y productividad de la empresa Comercializadora Mundo Gres del municipio de San Cayetano Norte de Santander", Universidad Libre de Colombia Seccional Cúcuta, Cúcuta, 2017

[6] M. Torres-Carrasco y F. Puertas, "La activación alcalina de diferentes aluminosilicatos como una alternativa al Cemento Portland: cementos activados alcalinamente o geopolímeros", $\boldsymbol{R e v}$. ing. constr. vol.32, n.2 pp.05-12, 2017. http:// dx.doi.org/10.4067/S0718-50732017000200001.

[7] R.A. García León, R. Bolívar León y E. Flórez Solano, "Validación de las propiedades físicomecánicas de bloques H-10 fabricados en Ocaña Norte de Santander y la región," Revista Ingenio, vol. 10, no. 1, pp. 17-26, junio 2016. Recuperado de: https://revistas.ufps.edu.co/ index.php/ingenio/article/view/2075/2025

[8] C. Bonilla Chiriví, D.C. González Vásquez y A. López Camacho, "Desarrollo de la resistencia a la compresión de un geopolímero obtenido a partir de residuos de ladrillo de arcilla" Universidad La Gran Colombia, 2017

[9] L. Reig, L. Soriano, M.V. Borrachero, J.Monzó and J. Payá, "Influence of calcium aluminate cement (CAC) on alkaline activation of red clay brick waste (RCBW), Cement and Concrete Composites, vol. 65, pp. 177-185, January 2016
[10] J. Sánchez-Molina, A. Sarabia-Guarin, y D.C. Alvarez-Rozo, "Evaluación de materias primas utilizadas en la fabricación de baldosas de gres en el sector cerámico de Norte de Santander (Colombia)", Respuestas, vol. 21, no. 2, pp. 48 -56 , jul. 2016

[11] J.F. Gelves-Diaz, J. Sánchez-Molina, y G. PeñaRodríguez, "Comportamiento de las arcillas del área metropolitana de Cúcuta sometidas a proceso de moldeo por extrusión", Respuestas, vol. 14, n. $^{\text {o }} 2$, pp. $32-38$, may 2016

[12] L. Reig, M.M. Tashima, M.V. Borrachero, J. Monzó, C.R. Cheeseman, J. Payá, "Properties and microstructure of alkali-activated red clay brick waste", Construction and Building Materials, vol. 43, pp. 98-106, June 2013

[13] R.A. Robayo, A. Mulford, J. Munera y R. Mejía, "Alternative cements based on alkaliactivated red clay brick waste", Construction and Building Materials, vol. 128, pp. 163-169, 2016

[14] N.R. Rakhimova , R.Z. Rakhimov, "Alkali - activated cements and mortars based on blast furnace slag and red clay brick waste", Materials and Design, vol. 85, pp. 324-331, 2015 